\title{
Collaborative and Infrastructure-less Vehicular Traffic Rerouting for Intelligent Transportation Systems
}

\author{
Ademar T. Akabane ${ }^{1}$, Edmundo R. M. Madeira ${ }^{1}$ (Co-advisor), \\ Leandro A. Villas ${ }^{1}$ (Advisor) \\ ${ }^{1}$ Institute of Computing, University of Campinas - Campinas, Brazil \\ takeo@lrc.ic.unicamp.br, \{edmundo, leandro\}@ic.unicamp.br
}

\begin{abstract}
This extended abstract provides an at-a-glance view of the main contributions of my Ph.D. work. The work aims to investigate and develop cuttingedge an infrastructure-less vehicular traffic management system in order to minimize vehicular traffic congestion and advance the state-of-the-art in intelligent transportation systems. The proposed solutions were widely compared with other literature solutions on different performance evaluation metrics. The evaluation results show that the proposed vehicle traffic management system is efficient, scalable, and cost-effective, which may be a good alternative to mitigate urban mobility problems.
\end{abstract}

\section{Introduction}

Traffic congestion is a daily occurrence for citizens living in large cities around the world. This problem tends to worsen with the economic and population growth in the urban centers. The increasing vehicular traffic demand may overwhelm the existing transport infrastructure, especially during rush hours [Akabane et al. 2017a, Akabane et al. 2020]. To improve on this issue, two immediate solutions come to mind: (i) the expansion of road infrastructure; or (ii) the amendment of the traffic management system. In the former solution, the cost of road infrastructure expansion is often impractical, due to financial and/or physical-space constraints. The latter solution, on the other hand, allows the use of already existing technologies, along with the new ones, to improve the efficiency of the traffic management system. This can be done without the need to invest in new road infrastructure. Due to the limitations mentioned in the first solution, significant research efforts have been directed toward the second one [Nellore and Hancke 2016, Wang et al. 2016, Akabane et al. 2019a, Akabane et al. 2019b, Akabane et al. 2018b].

For example, SCOOT ${ }^{1}$ and SCATS ${ }^{2}$ were the first two systems that employed information and communication technologies (ICT) for traffic management. The SCOOT and SCATS systems need a traffic operation center (TOC) that manages all traffic lights and optimizes the traffic light timings. Such systems, basically, during fixed time intervals, collect the real-time traffic information by induction loops that are installed underground of major urban roads. Using such information, the system can identify the vehicle flow on the induction loop area, thus adjusting the time cycle of traffic lights. To optimize the traffic light timings and control vehicles queuing in front of junctions, both systems need a TOC that manages all traffic lights. Another ICT service commonly used for congestion control is the vehicle navigation system. This type of system collects traffic information through the user's mobile devices, and the best known are Google $\mathrm{Maps}^{3}$,

\footnotetext{
${ }^{1} \mathrm{https}: / /$ trlsoftware.com/products/traffic-control/scoot/

${ }^{2}$ https://www.scats.com.au/

${ }^{3}$ https://maps.google.com
} 
TomTom $^{4}$, and Waze ${ }^{5}$. Thus, the users of these systems can monitor the current traffic conditions easily to plan their travel routes.

The current vehicles are equipped increasingly with a variety of computational resources, for example, sensors, cameras, and wireless communication devices to facilitate the utmost travel comfort and safety of drivers and passengers. Through the advancement of wireless communication technology, a new paradigm of wireless networks, known as vehicular ad-hoc networks (VANETs) [Akabane et al. 2015, Nellore and Hancke 2016, Wang et al. 2016], is emerging. Thus, VANETs can collect, process and share sensed data supporting various intelligent transportation systems (ITS) applications such as advanced traffic management systems and urban environment sensing. Thus, we firmly believe that VANETs can help deal with urban mobility problems. Due to the current global trend of urbanization, modern society is facing serious urban mobility problems, higher fuel prices, and an increase in $\mathrm{CO}_{2}$ emissions. In addition to that, with the constant increase in vehicular traffic on roads, existing traffic management solutions have become inefficient. In order to serve the increasing needs of transport systems, there is a need for ITS. Developing a sustainable ITS requires seamless integration and interoperability with emerging technologies such as VANETs.

\section{Objective}

The main objective of the thesis is to design, implement, and evaluate a collaborative and infrastructure-less system for vehicular traffic management. To achieve this goal, we need to answer the following questions:

- Many VANET-based traffic management systems were proposed to generate a global view of the road network, allowing the detection of all possible road traffic congestions. These systems need constant information exchange between the vehicle and the central server in order to obtain a global view of road traffic conditions. In this regard, it is known that if this information exchange is not well managed, it can lead to network overload.

Research Question \#1: How can we obtain a global view of road network topology without exchanging data between vehicles and the central server for traffic management purposes?

- High mobility of nodes is the main characteristic of VANETs. Therefore, identifying and selecting the best-located vehicles available at the right time and place for a given application task through inter-vehicle communications is a very challenging task. The best-located vehicle is defined as the importance of the car concerning the information flows that passes through it. On the other hand, once it is identified, it can be beneficial for a large number of services, such as those that spread the information flow through the network.

Research Question \#2: How can we dynamically identify the best-located vehicle among the candidate ones, in a distributed manner, to perform a given application task?

- It is known that the primary goal of the vehicle rerouting algorithm is to move vehicular traffic away from the congestion point. To this end, two main requirements for this type of algorithm in VANETs are expected: (i) to calculate alternative routes for each vehicle that can improve the vehicle's path and also maximize the global network efficacy; and (ii) to alert vehicles quickly so that they have

\footnotetext{
${ }^{4}$ https://www.tomtom.com

${ }^{5}$ https://www.waze.com
} 
enough time to compute a new route. To do this, collaborative route planning was proposed to answer the question below. It is worth mentioning that this type of planning takes into account the surrounding vehicles' routes to compute an alternative route.

Research Question \#3: Can collaborative route planning help effectively minimize traffic congestions without compromising scalability?

- Several systems have been proposed to deal with issues related to vehicular traffic management. Usually, their solutions include the integration of computational technologies such as vehicular networks, central servers, and roadside units. Most of them apply a hybrid approach, which means they still need a central entity (central server or roadside unit) and Internet connection to achieve their objectives. It is known that integrating different types of technologies increases the cost of developing systems and often making the implementation unfeasible.

Research Question \#4: Can infrastructure-less vehicular traffic management systems be as efficient as infrastructure approaches and also scalable and cost-effective?

\section{Main Contributions}

The main contributions of the thesis are a people-centric approach for vehicular traffic management, a comparative study on the egocentric and sociocentric betweenness measure in VANETs, a distributed system for information management and knowledge distribution, and collaborative and infrastructure-less vehicular traffic management. In summary, we have:

\section{Routing Protocol using Mobility Pattern}

This contribution concerns the proposal of a vehicle routing protocol. It is currently known that there are anonymized datasets concerning the mobility patterns of the drivers ${ }^{6,7,8}$ in the urban center. Based on that, we proposed a protocol that uses historical knowledge of mobility patterns of the drivers to obtain a global view of the road network situations, dealing directly with Research Question \#1. Our approach has two different stages: (i) - Offline, in which the historical data processing of the global view of the road network is performed in order to generate the mobility patterns; and (ii) - Online, in which vehicles in the route to congested roads are re-routed. The proposed protocol acts as a traffic monitoring system, having an overview of road networks without needing to periodically exchange information status between the central server and the vehicles. Simulation results have shown that such an approach could be a suitable alternative for traffic management.

\section{Vehicle Ranking Mechanism}

This contribution concerns the proposal of an innovative vehicle ranking mechanism called $V_{\text {rank }}$, dealing with Research Question \#2. It is known that Google's PageRank [Page et al. 1999] algorithm ranks the importance of webpages based on the number of web-links directed towards it. The general idea of PageRank relies on a graph where nodes are webpages and edges depict the links between them. Thereby, PageRank uses the link structure as an indicator of an individual page's importance in the structure of the World Wide Web relative to other pages. In general, the higher the number of links, the greater the importance of

\footnotetext{
${ }^{6} \mathrm{http}: / /$ kolntrace.project.citi-lab.fr/

${ }^{7} \mathrm{http}: / /$ www.vehicularlab.uni.lu/lust-scenario/

${ }^{8}$ https://crawdad.org/epfl/mobility/20090224/
} 
the webpage. The idea of $V_{\text {rank }}$ is to use the link structure of VANETs to compute the vehicle's score. To do this, we used the Egocentric Betweenness Metric. Betweenness is a measure of how often a node is located on the geodesic distance (shortest path) between other nodes in the network. It thus measures the importance to which the node can function as a point of control in the communication. Intuitively, the betweenness metric measures the control a node has over communication in the network. High betweenness value, thus implying that a node can reach other nodes on relatively short path or that a node lies on a considerable fraction of shortest paths connecting pairs of other nodes. Simulation results have demonstrated that by using the $V_{\text {rank }}$, it is possible to make the system scalable.

3. Collaborative Route Planning

This contribution consists of proposing, designing, and evaluating collaborative route planning to improve vehicular traffic management on urban road scenarios. Generally speaking, vehicles traveling in the congestion region collaborate by exchanging information about their alternate routes chosen that bypass the congestion. The idea here is that each vehicle plans its available alternative routes to the destination taking into account the alternative route information received from surrounding vehicles. Through this collaboration, each vehicle can create an awareness to which roads vehicles are being moved to, thus planning the most suitable alternative route and avoiding potential future congestion. Simulation results have shown that collaborative decision making is more efficient than selfish decision making in alternative routes planning, dealing with Research Question \#3.

4. Infrastructure-less Vehicular Traffic Management System

This contribution consists of proposing, implementing, and evaluating a collaborative and infrastructure-less vehicular traffic management system in the urban scenario. Such a system dealing with Research Question \#4. It is worth mentioning that such a system takes into account the contributions presented in Items 2 and 3 previously presented to achieve its goal. Simulation results have demonstrated that the proposed solution tends to be more scalable than infrastructure ones, and the collaborative routing strategy is more suitable in urban mobility management.

\section{Publications}

In the following sections, we present a list of publications produced from the thesis. The list is divided into journals, conference papers, and book chapter:

\subsection{Journals}

1. [Akabane et al. 2019]: Elsevier Ad Hoc Networks. [QUALIS A2 - Impact Factor: 3.490]. Under review;

2. [Akabane et al. 2020]: Elsevier Computer Communications. [QUALIS A2 - Impact Factor: 2.766];

3. [Akabane et al. 2019b]: Sensors (19)16, 3558. [QUALIS A1 - Impact Factor: 3.031];

4. [Akabane et al. 2018a]:. Sensors (18)8, 2731. [QUALIS A1 - Impact Factor: 3.031].

\subsection{Conferences}

1. [Akabane et al. 2019a]: Brazilian Symposium on Computer Networks and Distributed Systems (SBRC). [QUALIS A4]; 
2. [Akabane et al. 2018b]: IEEE Vehicular Networking Conference (VNC). [QUALIS A2];

3. [Akabane et al. 2018d]: Brazilian Symposium on Computer Networks and Distributed Systems (SBRC). [QUALIS A4];

4. [Akabane et al. 2018c]: IEEE Symposium on Computers and Communications (ISCC). [QUALIS A3];

5. [Akabane et al. 2017c]: IEEE Vehicular Technology Conference (VTC-Fall). [QUALIS A1];

6. [Nikolovski et al. 2017]: IEEE Symposium on Computers and Communications (ISCC). [QUALIS A3];

7. [Akabane et al. 2017b]: IEEE Network Computing and Applications (NCA). [QUALIS B1];

8. [Akabane et al. 2017a]: IEEE Global Communications Conference (GLOBECOM). [QUALIS A1];

9. [Santos et al. 2016]: IEEE Vehicular Technology Conference (VTC-Fall). [QUALIS A1].

\subsection{Book Chapter}

1. [Rodrigues et al. 2019]: Brazilian Symposium on Computer Networks and Distributed Systems (SBRC). [QUALIS A4].

\section{Main Results}

To overcome the limitations and costs of infrastructured ITS applications, we proposed the SOPHIA system. This system dealing with the problems associated with traffic congestion, in a distributed manner, and without jeopardizing its scalability. The main results can be summarized and were divided into two perspectives (or assessments), which are represented as follows, see Figures 1 and 2. All figures show the performance result in relation to the vehicle insertion rate. That is, for example, $20 \%$ means that only $20 \%$ of the total vehicles are inserted in the scenario for the simulation experiments, and so on. Each sub-figure is composed of two bar charts. The top one represents the numerical value of the assessed metric and the bottom one represents the gain with respect to EcoTrec.

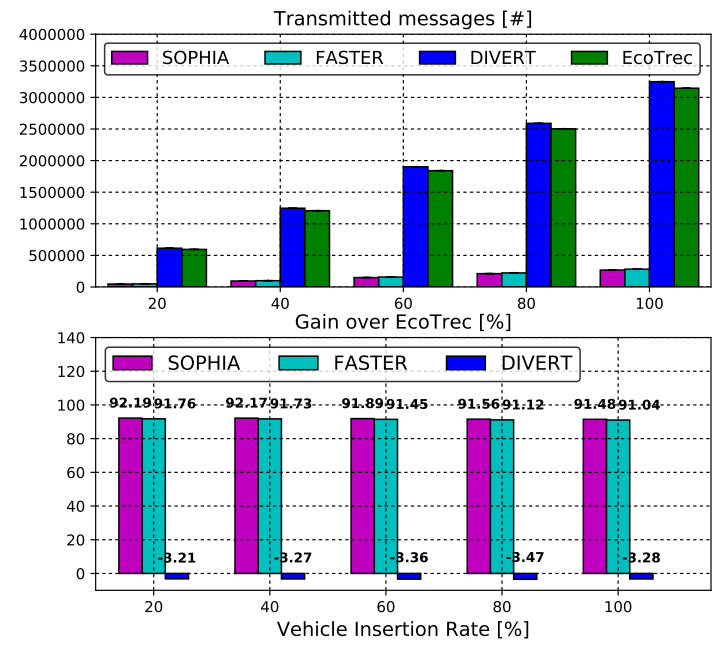

(a) Total of transmitted messages.
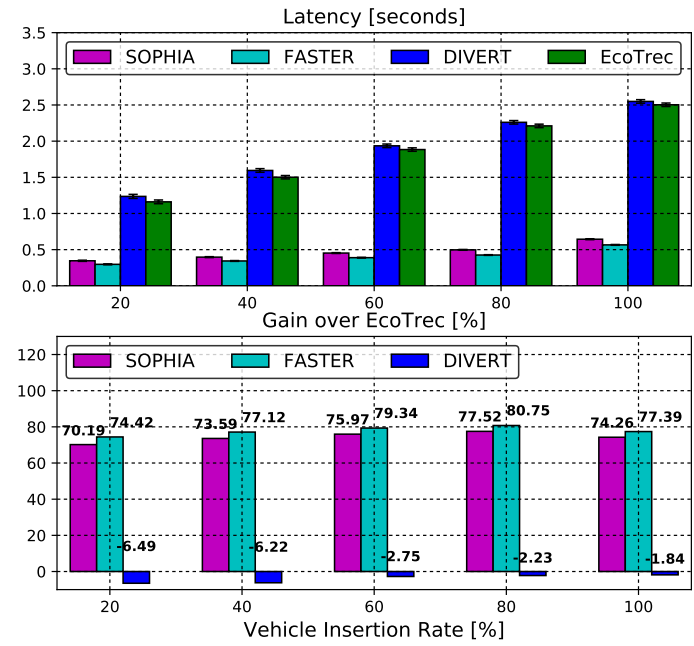

(b) Latency.

Figure 1. The results of the scalability assessment. 
Figure 1(a) displays the performance results of EcoTrec, DIVERT and FASTER systems according to the overhead metric. It is worth highlighting that EcoTrec and DIVERT, constantly need to exchange messages between the vehicles and the central server to reach their purposes. Due to this strategy, it is possible to observe that both have a higher average rate of messages transmitted in relation to FASTER and SOPHIA (both infrastructure-less). Another determining factor for this high rate, for both systems, is the absence of a broadcast suppression mechanism during the message distribution process. By examining carefully, it is possible to notice that DIVERT has a slightly higher transmission rate than EcoTrec. This is because DIVERT, in addition to communicating with the central server, implements a collaborative routing mechanism when choosing an alternative route. It is worth mentioning that such a mechanism contributes to vehicular traffic management and this contribution will be discussed in the following paragraphs. Both FASTER and SOPHIA apply vehicle selection techniques for the extraction of knowledge. FASTER segments the scenario into several sub-regions and in each of them one vehicle for knowledge extraction is selected. However, SOPHIA applies a dynamic clustering approach to select the most appropriate vehicle. The dynamic clustering is more appropriate, in this case, as it does not need to segment the scenario for the vehicle selection. It should also be mentioned that both FASTER and SOPHIA applies a mechanism to deal with the broadcast storm problem. Additionally, both have similar performance and they can drastically reduce the total amount of transmitted messages, more than $91 \%$ decrease in comparison with DIVERT and EcoTrec, as shown in Figure 1(a) (bottom).

Another metric evaluated is the transmission latency in relation to the vehicle insertion rate, Figure 1(b). In both, the infrastructural and distributed approaches, as vehicle insertion rates increase the latency also increases, as expected. This is because raising the number of vehicles in the simulations increases the network overhead caused by the exchange of messages. However, FASTER and SOPHIA have the lowest latencies compared to other systems analyzed. Comparing numerically, the mean delay of the SOPHIA, FASTER, DIVERT, and EcoTrec systems are around 0.48, 0.42, 1.93, and $1.87 \mathrm{~s}$, respectively. Comparing SOPHIA and FASTER systems between each other, we can observe that the FASTER system has a slight reduction in latency. This is because the knowledge is extracted in several sub-regions, thus delivering it more rapidly to vehicles, however with a data transmission greater than SOPHIA, see Figure 1(a) (bottom). Both SOPHIA and FASTER have an average reduction above $74 \%$ compared to the EcoTrec and DIVERT systems, as shown in the Figure 1(b) (bottom).
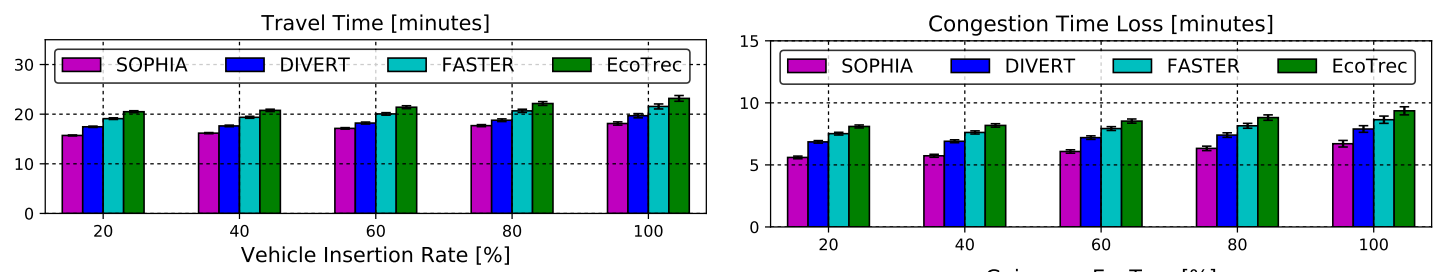

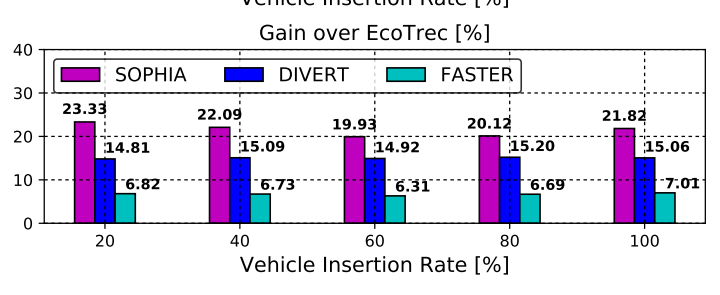

(a) Travel time.

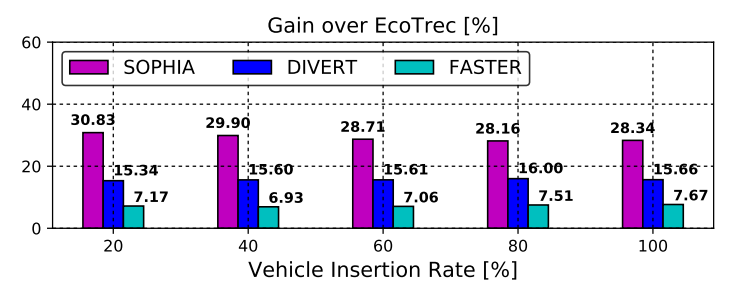

(b) Congestion.

Figure 2. The results of the traffic management assessment. 
Figure 2(a) shows the result of the average travel time for all insertion rates. From the figure, it is possible to notice that the higher the vehicle insertion rate, the longer the average travel time for all solutions analyzed. This behavior is expected since, at high rates the roads become denser, leading to the occurrence of congestion. Among all solutions analyzed, EcoTrec system has the longest average travel time, around $22 \mathrm{~min}$. EcoTrec choices an alternative route by the path that emits the lowest $\mathrm{CO}_{2}$ rate until the trip destination. Differently, the FASTER system selects a selfish route based on the probabilistic $k$-shortest path. This strategy has a gain of $6.71 \%$ against EcoTrec. Another approach is taken by the DIVERT system, where the vehicles calculate an alternative route collaboratively. In this approach, it is possible to notice a reduction in the mean travel time around $15 \%$ and $8.3 \%$, compared to EcoTrec and FASTER, respectively. The SOPHIA system applies collaborative routing, such as DIVERT. Even so, it overcomes DIVERT in this metric, due to the low network overhead. As mentioned before, DIVERT has a higher overhead, so many messages arrive corrupted at the recipients. Analyzing numerically, SOPHIA achieves a mean reduction of $6.46 \%, 14.75 \%$, and $21.46 \%$ compared to DIVERT, FASTER, and EcoTrec, respectively, see Figure 2(a) (bottom).

Another metric considered was the time lost in congestion, Figure 2(b). All evaluated systems apply some vehicle rerouting mechanism after congestion detection. It is important to emphasize that systems that implement collaborative routing outperform the selfish one. This can be observed in Figures 2(a) and 2(b). To demonstrate them numerically, DIVERT achieves a time reduction of approximately $7.87 \%$ and $15.14 \%$ over FASTER and EcoTrec, respectively. While SOPHIA reaches approximately $21.92 \%$ and $29.18 \%$ compared to FASTER and EcoTrec, respectively, see Figure 2(b) (bottom). As mentioned earlier, the SOPHIA system has a lower overhead compared to DIVERT. Therefore, this fact contributes to the information reaching the largest number of participants thus contributing to improving traffic management efficiency.

\section{Final Remarks}

This extended abstract sums up the contributions of the thesis in [Akabane et al. 2019c] dealing with the challenges of building a reliable and scalable infrastructure-less vehicular traffic management system. The main contributions of the thesis are a people-centric approach for vehicular traffic management, a comparative study on the egocentric and sociocentric betweenness measure in VANETs, a distributed system for information management and knowledge distribution, and collaborative and infrastructure-less vehicular traffic management. The knowledge produced during research has been featured in several top-tier venues in the area, either in terms of scientific publications or in short course.

\section{References}

Akabane, A., Immich, R., Pazzi, R., Madeira, E., and Villas, L. (2018a). Distributed egocentric betweenness measure as a vehicle selection mechanism in vanets: A performance evaluation study. Sensors, 18(8):2731.

Akabane, A. T., Gomes, R. L., Pazzi, R. W., Madeira, E. R., and Villas, L. A. (2017a). Apolo: A mobility pattern analysis approach to improve urban mobility. In GLOBECOM 2017-2017 IEEE Global Communications Conference, pages 1-6. IEEE.

Akabane, A. T., Immich, R., Bittencourt, L. F., Madeira, E. R., and Villas, L. A. (2020). Towards a distributed and infrastructure-less vehicular traffic management system. Elsevier Computer Communications. 
Akabane, A. T., Immich, R., Madeira, E., and Villas, L. (2019a). Aplicando redes sociais veiculares para aprimorar o gerenciamento da mobilidade urbana. In Anais do XXXVII Simpósio Brasileiro de Redes de Computadores e Sistemas Distribuídos, pages 1070-1083. SBC.

Akabane, A. T., Immich, R., Madeira, E. R., and Villas, L. A. (2018b). imob: An intelligent urban mobility management system based on vehicular social networks. In 2018 IEEE Vehicular Networking Conference (VNC), pages 1-8. IEEE.

Akabane, A. T., Immich, R., Pazzi, R. W., Madeira, E. R., and Villas, L. A. (2018c). Trusted: A distributed system for information management and knowledge distribution in vanets. In 2018 IEEE Symposium on Computers and Communications (ISCC), pages 1-6. IEEE.

Akabane, A. T., Immich, R., Pazzi, R. W., Madeira, E. R. M., and Villas, L. A. (2019b). Exploiting vehicular social networks and dynamic clustering to enhance urban mobility management. Sensors, 19(16):3558.

Akabane, A. T., Madeira, E. R., and Villas, L. A. (2019c). Collaborative and infrastructure-less vehicular traffic rerouting for intelligent transportation systems.

Akabane, A. T., Pazzi, R. W., Madeira, E. R., and Villas, L. A. (2017b). Applying egocentric betweenness measure in vehicular ad hoc networks. In 2017 IEEE 16th International Symposium on Network Computing and Applications (NCA), pages 1-4. IEEE.

Akabane, A. T., Pazzi, R. W., Madeira, E. R., and Villas, L. A. (2017c). Modeling and prediction of vehicle routes based on hidden markov model. In 2017 IEEE 86th Vehicular Technology Conference (VTC-Fall), pages 1-5. IEEE.

Akabane, A. T., Pazzi, R. W., Madeira, E. R., and Villas, L. A. (2018d). Sistema distribuído para gerenciamento de informação e distribuição de conhecimento em redes veiculares. In Anais do XXXVI Simpósio Brasileiro de Redes de Computadores e Sistemas Distribuídos. SBC.

Akabane, A. T., Villas, L. A., and Madeira, E. R. M. (2015). An adaptive solution for data dissemination under diverse road traffic conditions in urban scenarios. In 2015 IEEE wireless communications and networking conference (WCNC), pages 1654-1659. IEEE.

Nellore, K. and Hancke, G. P. (2016). A survey on urban traffic management system using wireless sensor networks. Sensors, 16(2):157.

Nikolovski, T., Pazzi, R. W., Akabane, A. T., and Villas, L. A. (2017). Efficient encounter-based event dissemination protocol (e-bed) for urban and highway vehicular ad hoc networks. In 2017 IEEE Symposium on Computers and Communications (ISCC), pages 1085-1090. IEEE.

Page, L., Brin, S., Motwani, R., and Winograd, T. (1999). The pagerank citation ranking: Bringing order to the web. Technical report, Stanford InfoLab.

Rodrigues, D. O., Santos, F. A., Geraldo Filho, P. R., Akabane, A. T., Cabral, R., Immich, R., Junior, W. L., Cunha, F. D., Guidoni, D. L., Silva, T. H., et al. (2019). Computação urbana da teoria a prática: Fundamentos, aplicações e desafios. arXiv preprint arXiv:1912.05662.

Santos, F. A., Akabane, A. T., Yokoyama, R. S., Loureiro, A. A., and Villas, L. A. (2016). A roadside unit-based localization scheme to improve positioning for vehicular networks. In 2016 IEEE 84th Vehicular Technology Conference (VTC-Fall), pages 1-5. IEEE.

Wang, S., Djahel, S., Zhang, Z., and McManis, J. (2016). Next road rerouting: A multiagent system for mitigating unexpected urban traffic congestion. IEEE Transactions on Intelligent Transportation Systems, 17(10):2888-2899. 